\title{
Research of adaptive middleware based on context awareness
}

\author{
Jing Zhang ${ }^{1, a^{*}}$, Guangyu Wang ${ }^{1, b}$ and Xiaoli Wang ${ }^{1, c}$ \\ ${ }^{1}$ Aviation University of Air Force, Changchun, China 130022 \\ a524402684@qq.com, ${ }^{b} 1803097835 @ q q . c o m,{ }^{\circ} 524402684 @ q q . c o m$
}

\begin{abstract}
Keywords: context aware; Pervasive computing; Adaptive middleware; Adaptive strategies
Abstract. The limitation of mobile environment and equipment, brings the new challenges for pervasive computing. This paper analyzes the problems existing in the traditional middleware technology in pervasive computing, puts forward the necessity of adaptive mechanisms in middleware implementation. Through the analysis of the current context aware systems, which effectively applied to middleware, put forward the suitable for pervasive computing, and capable of context aware adaptive middleware model. At the same time, the method of context awareness and adaptive strategy are discussed.
\end{abstract}

\section{Introduction}

In recent years, with the development and application of embedded technology, portable mobile devices are emerging in an endless stream, They have the characteristics of miniaturization, intelligence and embedded. Meanwhile, the emergence and maturity of the wireless network technology has brought great convenience to the interconnection of these computing devices, and promote the reform of the computing model. The emergence of pervasive computing provides a new way for people to study, and it also puts forward new challenges for the development of heterogeneous environment. However, the pervasive environment with heterogeneous, distributed and dynamic. Pervasive computing terminals generally have tracking and re positioning of the ability, and the ability to adapt to the changing external environment and to keep the real mutual communication. However, traditional middleware technology is difficult to meet the dynamics in pervasive environment, will encounter such as adaptive performance is poor, heavyweight, and lack of flexibility. This paper in the middleware, pervasive environment, context aware research based on the proposed research on adaptive middleware for pervasive computing, provides a good platform for pervasive environment development.

\section{Middleware and pervasive computing}

Middleware is a distributed software layer, abstract the complexity and heterogeneous nature of the underlying distributed environment, by providing a simple, consistent and integrated distributed programming environment, simplify the design and development of distributed application. The pervasive computing terminal processing capacity and limited resources, and support for pervasive computing middleware must be lightweight and can't take too much system resources. At the same time, due to the intermittent nature wireless network bandwidth of regular variation and network connection, pervasive computing middleware systems need to support context aware, in order to adapt to the changing context, such as network bandwidth, memory and computing power. Traditional middleware specific implementation details to the user and system application developers is not visible, although these middleware technology successfully construct the traditional distributed systems, but they are applied to mobile environment, has exposed the serious defects. In pervasive computing environment to the user hiding all details of the implementation is not appropriate, the application might contain some information that could help to improve the efficiency of the middleware. In addition, such "black box" system is unavoidably a heavyweight, strategies and mechanisms of middleware embedded in pervasive computing does not apply. 


\section{Adaptive middleware}

In pervasive environment, the system resource is changed, so the application must have adaptive ability, through the different perception of context changing their behavior, so as to for users provide stable and reliable performance, the maximum extent to meet the user's needs. Therefore, we have put the adaptive mechanism in middleware layer. It is not only convenient for application development, and increase the adaptability of the application.According to the adaptive middleware requirements of the context aware and adaptive middleware features, we propose a can adapt to environmental changes of adaptive middleware can very good solution to the problem. According to different functions, we will adaptive middleware is divided into three modules: context aware, adaptive strategy and adaptive implementation body. The structure diagram shown in figure 1.

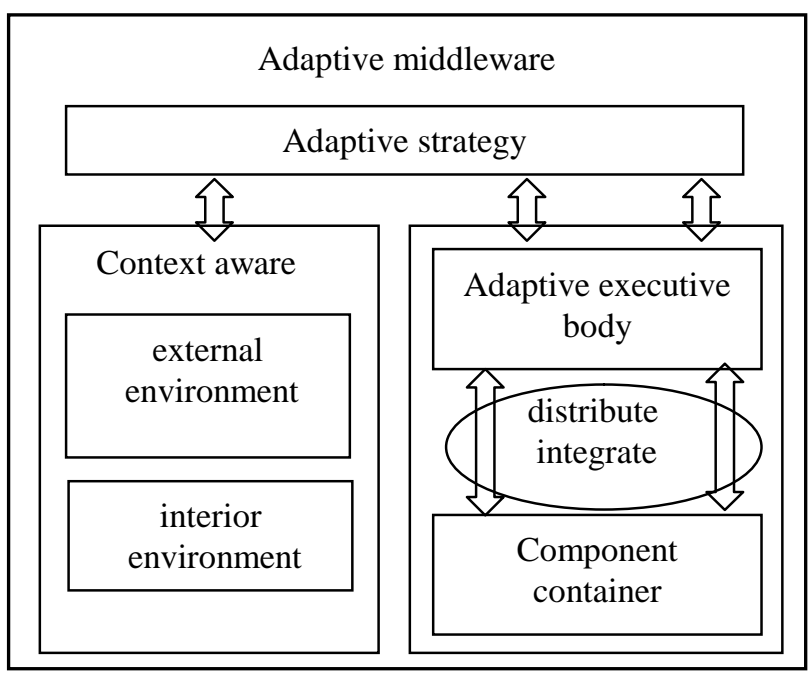

Fig. 1 Structure of adaptive middleware

\section{Context aware}

In this model, context awareness is divided into two aspects: the perception of external environment and the perception of internal resources.

(1) the perception of external environment.

It is mainly responsible for the form of equipment, context, physical, environmental and other computing environment management, coordination and scheduling, shielding calculation environment complexity, diverse and dynamic, adaptive layer provides a unified application interface. Context aware general including following several parts: definition of context and said, context and the storage and management of context acquisition. According to its function, we will perceive the external environment for context aware and evolutionary context, a context fusion device and the application program interface of four levels, as shown in Figure 2.

Context awareness: the original context is collected from a wide range of distributed sensors and is processed to separate the context aware from the actual use.

Context evolution: original context is fuzzy, imprecise, and contains the conflict, which requires the context evolution. Whose purpose is to shield the sensor differences, standardization of context information and context reasoning and fusion, high-level context required for a variety of applications.

Context fusion device: context aware computing is mainly reflected, including to the environment sensing and triggering, interoperability, and adaptive strategy, self configuration technology. Spontaneous interoperation can be removed from the user intervention, the adaptive strategy can support handwriting, voice input, so that it can be arbitrary switching.

Application program interface: the main responsibility to provide program development interface, so that developers can easily and quickly use. 


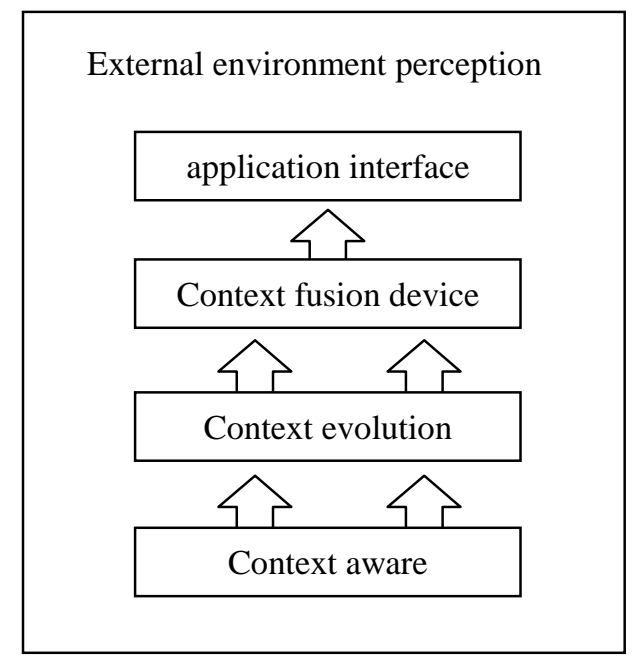

Fig. 2 External environment perception hierarchy diagram

(2) the perception of internal resources.

perception is mainly completed through the reflection mechanism, it through the mechanism of reflection components can get at run time the component interface connection information, components running status information, components between the logical dependencies, components of a resource consumption and the injection component information etc., introspection is mainly responsible for examine the current state of the internal resources to achieve.

\section{Adaptive executive body}

Adaptive executive body is the core of adaptive middleware and its basic unit is component containers in various autonomous components. Components can be a combination of one or a plurality of components, to meet the dynamic changes of context. Components of the assembly is the adaptive executive body, for the upper application provides services with adaptive ability.

(1) adaptive components

in order to be able to dynamically allocate and combination, the adaptive component should have the following points.

$\ddot{y}$ More attention is focused on separation, using AOP's focus on separation methods, providing functional and non functional interfaces.

$\ddot{y}$ With good message mechanism, the communication between the components is real-time, good news to send and receive to provide a reliable guarantee.

$\ddot{y}$ Self described interface can be used to know the function logic, running state and resource utilization of the components.

$\ddot{y}$ In support of heterogeneous, distributed environment, there are different levels of heterogeneity, the adaptive component must be able to support the heterogeneous environment.

(2)adaptive executive body

The executor of the dynamic allocation and adaptive. It is strategy implementation, with high intelligence and autonomy. Intelligent adaptive is a logical entity, it is logical combinations of component. At the same time self adjust and update. It can in adaptive strategy under the guidance, automatic the component allocation and combination.

\section{Adaptive strategies}

Adaptive strategy is to realize the premise of dynamic configuration. It is mainly responsible for the content of the two aspects. First, through context awareness mechanism of external environment and 
internal resources analysis, in order to ensure the system is safe and reliable and, adaptive strategy. Second, adaptive evaluation result is good or bad, if the self configuration results cannot satisfy the application demand and no better than previous configuration, the adaptive strategy should to return the last configuration. This will ensure that the system will be run worse than the original.

\section{Implementation process of dynamic configuration}

The dynamic configuration of adaptive mobile middleware is as follows:

Step 1, the context aware mechanism to perceive the external environment changes, identify the context of the current environment, and compare the different from the original context.

Step 2, the context aware mechanism is used to obtain the current utilization status of internal resources.

Step 3, according to the analysis of the external environment and internal resources of the context aware mechanism, select the appropriate strategy.

Step 4, according to the selected strategy, the application will be divided into different components according to function, the completion of the distribution and integration of components, the formation of a complete adaptive agent.

\section{Summary}

As people's need for intelligent and autonomy, adaptive middleware for pervasive computing technology will become the development trend of future research. We analyzed the adaptive middleware requires a few things, gives an adaptive architecture of the middleware based on context awareness. The next step work consideration in the design of adaptive middleware to join ontology, can better support context awareness, increase of the dynamic characteristics of pervasive computing and adaptability.

\section{Acknowledgements}

This work was financially supported by the social science research project of Jilin Provincial Department of Education([2016]494).

\section{References}

[1] Zhou Yu,Cao Jiannong. A middleware support for agent-based applicationmobility in pervasive environments[J].Distributed Computing Systems Workshops, 2007, 22(29): 9.

[2] Chen G,Kotz D.A Surey of Context-Aware Mobile Computing Research[D].Hanover: Department of Computer Science of Dartmouth College, 2000.

[3] Kamran Sheikh,Maarten Wegdam, Marten van Sinderen. Middleware support for quality of context in pervasive context-aware systems [J]. Pervasive Computing and Communications Workshops, 2007, 19(23): 461

[4] J.Heer,A.Newberger,C.Beckmann,et al.Context- aware distributed queries[J]. Lecture Notes in Computer Science,2003,1: 140- 148. 